\title{
Chapter 2 \\ Context Effects in Bilingual Sentence Processing: Task Specificity
}

\author{
Eva Van Assche, Wouter Duyck, and Robert J. Hartsuiker
}

\begin{abstract}
This chapter provides an overview of bilingualism research on visual word recognition in sentence context and relates this work to task-specific context factors. Many studies examining bilingual word recognition out-of-context have shown that words from both languages become activated when reading in one language (i.e., language-nonselective lexical access). A recent research line investigated whether presentation of words in a sentence context, providing a language cue and/or semantic constraint to restrict lexical access to words in the target language, modulates this language-nonselective activation. Recent lexical decision, translation, naming, and eye-tracking studies suggest that the language of the sentence context cannot restrict lexical access to words of the target language. Eye-tracking studies revealed that semantic constraint of a sentence does not necessarily restrict language-nonselective access, although there is evidence that it has a relatively late effect, and that it affects language-nonselective activation in lexical decision, translation, and naming studies.
\end{abstract}

\section{Introduction}

A fundamental issue in the domain of bilingualism concerns the organization of the bilingual language system. One viewpoint is that bilinguals have two separate lexicons that can be accessed selectively so that they can effectively function like monolinguals. Another viewpoint is that they have an integrated lexicon containing all words in both languages that can be accessed in a language-nonselective way. In the last decade, more and more researchers have provided evidence for this latter view.

E. Van Assche, Ph.D. ( $ه) \bullet$ W. Duyck, Ph.D. • R.J. Hartsuiker, Ph.D.

Department of Experimental Psychology, Ghent University,

Henry Dunantlaan 2, Ghent 9000, Belgium

e-mail: eva.vanassche@ugent.be; Wouter.Duyck@ugent.be; Robert.Hartsuiker@ugent.be 
It has become clear that lexical representations of the first language (L1) are accessed when bilinguals are reading single words in their second language (L2; Dijkstra, Grainger, \& Van Heuven, 1999; Duyck, 2005; Jared \& Kroll, 2001; Lemhöfer \& Dijkstra, 2004) and vice versa (e.g., Duyck, 2005; Van Hell \& Dijkstra, 2002). Only recently has this question been addressed in relation to how context and the semantic constraint provided by a sentence might modulate this languagenonselective activation for single word reading (e.g., Schwartz \& Kroll, 2006; Van Assche, Duyck, Hartsuiker, \& Diependaele, 2009; Van Hell \& De Groot, 2008). In the present chapter, we provide an overview of single-word and sentence processing studies on bilingual visual word recognition and discuss how task characteristics might modulate the results.

\section{Bilingual Visual Word Recognition Out-of-Context}

To investigate whether bilinguals activate words in both languages or only in the contextually relevant language when reading, the processing of words that are similar across languages is often compared to the processing of language unambiguous words. For instance, cognates are translation equivalents with a similar or equal spelling across languages (e.g., Spanish-English papel-paper). These words are typically read faster than noncognates that have no orthographic overlap across languages (e.g., silla-chair). This cognate facilitation effect is typically explained by assuming language-nonselective activation in which words from both languages are activated in parallel (e.g., Dijkstra, Miwa, Brummelhuis, Sappelli, \& Baayen, 2010; Dijkstra \& Van Heuven, 2002). The presentation of a word in one language co-activates orthographically, phonologically, and semantically similar words in the other language. Since cognates share orthographic, phonological, and semantic information across languages, whereas noncognates only share semantic information, the convergent cross-lingual activation of these representations speeds up the recognition of cognates as compared to noncognates. The cognate facilitation effect has been consistently found for word reading in L2 (e.g., Caramazza \& Brones, 1979; Dijkstra et al., 1999; Lemhöfer \& Dijkstra, 2004; Lemhöfer, Dijkstra, \& Michel, 2004) and even for word reading in L1 (e.g., Van Hell \& Dijkstra, 2002).

Van Hell and Dijkstra (2002) used an L1 (Dutch) lexical decision task to investigate whether knowledge of a second language influences native-language reading. In this task, which is the most frequently used experimental task to study crosslingual interactions in bilingual processing, bilinguals see letter strings on a computer screen and they have to decide as quickly and as accurately as possible whether a presented letter string is a real word (e.g., blouse) or not (e.g., flouse), in English, for example. Van Hell and Dijkstra tested reading in L1 in two groups of DutchEnglish-French trilinguals: one group was highly proficient in English and relatively low in their proficiency in French, and the other group was highly proficient in both English and French. The stimuli were L1-L2 cognates (e.g., Dutch hamer: hammer in English: marteau in French), L1-L3 cognates (e.g., Dutch citroen: lemon in 
English; citron in French) or matched control words (e.g., Dutch kelder: basement in English: cave in French). These three groups of Dutch (L1) words were matched for word length, word frequency, and number of orthographic neighbors (i.e., words differing by a single letter from the target such as snow, an intralingual neighbor of slow) in Dutch (Coltheart, Davelaar, Jonasson, \& Besner, 1977) because these factors have been shown to significantly influence word processing (e.g., New, Ferrand, Pallier, \& Brysbaert, 2006; Segui \& Grainger, 1990). This matching ensured that any observed differences in processing between cognates and noncognates could be attributed to the difference in cross-lingual overlap between cognates and noncognates and not to any uncontrolled stimulus characteristics. For both groups of trilinguals, the results showed a cognate facilitation effect for L1-L2 cognates. There was also an effect for L1-L3 cognates, but only for the participants who were highly proficient in both English (L2) and French (L3). Apparently, the occurrence of cross-lingual activation in L1 reading required a certain level of proficiency in L2 and L3. Nevertheless, these results show that even a second or third language gets activated strongly enough to influence native-language word processing.

Other evidence for dual-language activation comes from studies investigating the recognition of interlingual homographs (i.e., words that have the same orthographic form in both languages but have a different meaning; e.g., English red: net in Spanish). However, in contrast to the consistent replication of cognate facilitation effects, studies using homographs have yielded mixed effects depending on task requirements, stimulus list composition, and relative frequency of the homographs in the two languages (e.g., Dijkstra et al., 1999; Dijkstra, Timmermans, \& Schriefers, 2000; Dijkstra, Van Jaarsveld, \& Ten Brinke, 1998). Dijkstra et al. (1998) tested Dutch-English bilinguals in three lexical decision experiments. In Experiment 1, they performed an L2 (English) lexical decision task including interlingual homographs (e.g., English room: cream in Dutch) and monolingual control words that have no cross-lingual overlap (e.g., Dutch-English stoel-chair). Reaction times to homographs did not differ from monolingual controls suggesting that the Dutch reading of the homograph did not influence English word recognition. However, in Experiment 2, Dutch nonhomographic filler words were included in the English lexical decision task. Participants were instructed to respond with "yes" to English words (homographs and controls) and to respond with "no" to non-English words (Dutch words and nonwords). In this experiment, homographs were responded to more slowly than monolingual controls. The presence of Dutch words as nonwords might have boosted activation in the L1 lexicon, leading to stronger interference effects for homographs. In Experiment 3, the same stimuli were presented in a generalized lexical decision task in which a "yes" response had to be given to a word in either language. Under these task requirements, homographs were processed faster than monolingual control words, indicating that the fact that a homograph is a word in both languages speeds up its reaction time relative to controls. It seems that bilinguals then react to the fastest available representation in either language, leading to faster processing of homographs as compared to monolingual control words. Dijkstra et al. (1998) nicely illustrated that cross-lingual interactions and word reading can differ depending on task characteristics. 
In another study, Dijkstra et al. (1999) showed that the null results for interlingual homographs in Dijkstra et al.'s (1998) Experiment 1 could be clarified by distinguishing the orthographic and phonological overlap components of the homographs. Interlingual homographs have the same orthography in both languages but they can differ in the degree of phonological overlap. To investigate the effect of phonological overlap in interlingual homograph processing, they included English homographs that were either pronounced very similarly to Dutch words (e.g., English pet; cap in Dutch), or that were pronounced very differently to Dutch words (e.g., English glad; slippery in Dutch). The results showed that the processing of homographs with no phonological overlap was facilitated. This suggested that the facilitative influence of orthographic overlap and the inhibitory influence of phonological overlap led to the null effects in Dijkstra et al. (1998) and highlighted the importance of controlling for phonological similarity in the homograph stimuli.

To summarize, these studies on cognate and homograph word processing indicate that bilinguals cannot effectively function like monolinguals and that both languages interact and influence word recognition. Ever since, many studies have provided evidence for the viewpoint of language-nonselective activation of words in the two languages (e.g., Dijkstra et al., 2010; Dijkstra \& Van Heuven, 2002; Van Assche, Duyck, \& Hartsuiker, 2012). Does this language-nonselective activation similarly apply for bilinguals reading in context? Recently, studies began to test the ecological validity of the experiments presenting words out-of-context. After all, people rarely read lists of isolated words, but instead, words are embedded in meaningful sentences. It is possible that the presentation of words in a sentence context restricts lexical activation to words of the target (sentence) language only or allows for earlier language selection during lexical access. Indeed, studies in the monolingual domain have shown that semantic and syntactic restrictions imposed by a sentence context are used to speed up recognition of upcoming words (e.g., Schwanenflugel \& LaCount, 1988; Stanovich \& West, 1983). The question now is whether these monolingual sentence context effects generalize to bilingual sentence processing. Altarriba, Kroll, Sholl, and Rayner (1996) were the first to investigate word recognition in mixed-language sentences; a number of other studies investigating more natural unilingual sentence reading were carried out more recently (e.g., Duyck, Van Assche, Drieghe, \& Hartsuiker, 2007; Schwartz \& Kroll, 2006; Van Assche et al., 2009; Van Hell \& De Groot, 2008).

\section{Bilingual Visual Word Recognition in Sentences}

Studies of bilingual word recognition in sentences have used several different tasks to investigate how sentence context might modulate the cross-lingual activation effects observed in single-word studies. Different tasks may tap into different processes and may consequently lead to different result patterns related to the time course of word processing. In the following sections, we first discuss research using tasks requiring an overt response such as lexical decision or naming tasks, before presenting research using more natural reading tasks such as eye-tracking. 


\section{Reading Tasks Requiring Overt Responses}

One of the first investigations on bilingual word recognition in sentences is presented in Van Hell and De Groot (2008). Proficient Dutch-English bilinguals performed an L2 (English) lexical decision task or a translation task in forward (from L1 to L2) or in backward direction (from L2 to L1). The critical stimuli were cognates (e.g., Dutch-English: kapitein-captain) and matched control words (e.g., rok-skirt) presented out-of-context or preceded by a low- or high-constraint L2 sentence context. The sentence constraint manipulation allowed testing whether merely presenting words in a low-constraint sentence is sufficient to restrict lexical access to words of the target language or whether only a semantically constraining sentence can direct lexical access to the language of the sentence. Sentence completion ratings and plausibility ratings ensured that mean production probabilities did not differ for cognates and controls.

Experiment 1 investigated whether a meaningful sentence context and a semantically constraining sentence can guide lexical access to words of the target language and modulate the cognate facilitation effect. Dutch-English bilinguals were presented with an L2 (English) sentence context in which the target word was omitted (e.g., A green ___ and a yellow banana lay on the fruit dish). After the sentence context disappeared from the computer screen, the target (e.g., apple) for L2 lexical decision was presented. A control condition also presented target words out-ofcontext. The results showed that cognates were processed faster than controls when presented out-of-context. Cognate facilitation remained after reading a lowconstraint sentence context, but not after a high-constraint sentence context. This finding suggests that the semantic constraint of a sentence, but not the linguistic context and language cue provided by a sentence, can restrict cross-lingual activation effects.

Experiments 2 and 3 investigated how contextual information influences the translation of words. Sentences were presented as a whole with the target omitted (Experiment 2) or were presented word-by-word (Experiment 3). The target's translation had to be spoken out loud. In both experiments, results were comparable across the two translation directions (from L1 to L2 and from L2 to L1): cognate facilitation effects observed out-of-context remained in the presence of a lowconstraint sentence, but were strongly reduced when the words were presented after a semantically constraining sentence. The results of the lexical decision and translation tasks suggest that the feature restrictions imposed by a high-constraint, but not a low-constraint sentence, delineate the lexical and conceptual information of the upcoming words.

Schwartz and Kroll (2006) observed similar cognate results for word production in two sentence context experiments with highly proficient Spanish-English bilinguals living in a bilingual community and intermediate proficient SpanishEnglish bilinguals living in a monolingual community. They presented EnglishSpanish cognates (e.g., piano), interlingual homographs (e.g., fin), and monolingual control words in L2 (English) low- and high-constraint sentences. The sentences were 
presented word by word using a rapid serial visual presentation task (RSVP) and participants had to name the target word (printed in red) as quickly as possible (e.g., high-constraint cognate sentence: Before playing, the composer first wiped the keys of the piano at the beginning of the concert; high-constraint control sentence: Before the test, the student looked for some paper and a sharp pencil to write with). Cognate facilitation was observed in low-constraint sentences, but not in high-constraint ones. No reaction time differences were found for homographs and controls in either low- or high-constraint sentences, but bilinguals of intermediate proficiency made more errors than highly proficient ones, especially in lowconstraint sentences. Although the results for homographs were somewhat inconclusive, the results for cognate processing show that the semantic constraint of a sentence can restrict cross-lingual activation effects for both intermediate and highly proficient bilinguals.

A semantic priming study by Elston-Güttler, Gunter, and Kotz (2005) also investigated homograph processing in an L2 sentence context and tested how a more general language context may influence cross-lingual activation effects. To this end, German-English bilinguals saw either a German or English movie prior to the experiment, boosting L1 or L2 activation. Additionally, Elston-Güttler et al. tested how these language context effects change over time by analyzing the first and second halves of the experiment. German-English homographs (e.g., gift: poison in German) or control words (e.g., shell) were presented at the end of a relatively low constraining sentence (e.g., The woman gave her friend a pretty gift vs. The woman gave her friend a pretty shell). The sentence was then replaced by a target word for L2 (English) lexical decision (poison). If the L1 influences the L2 during word recognition, reading the homograph gift should influence subsequent processing of the related word poison. Targets were recognized faster after the related homograph sentence than after the unrelated control sentence, but only in the first half of the experiment and only for participants who saw a German movie prior to the experiment, boosting L1 activation. This semantic priming effect of the targets and their related homographs was also present in the recordings of event-related potentials in the modulation of the N200 and N400 components. The N200 component has been linked to word access and/or orthographic processing (e.g., Bentin, MouchetantRostaing, Giard, Echalier, \& Pernier, 1999). Elston-Güttler et al. suggested a translational word form link between gift-poison so that reading the prime gift leads to faster lexical access of the target poison. The N400 component has been linked to semantic integration processes (Brown \& Hagoort, 1993) suggesting that the target poison was easier to integrate and resulted in less negative N400 amplitude after the related prime gift than after the unrelated prime shell. This study showed that sentence context can eliminate the activation of the nontarget L1 homograph representation and that this effect is very sensitive to language context. Semantic priming effects were only observed when L1 activation was boosted prior to the experiment and only in the first half of the experiment. This suggests that context effects such as activating an L1 or L2 prior to the experiment can influence word recognition and that the bilingual language system can quickly zoom into the L2 processing context. 
These homograph studies did not distinguish word class overlap, and Baten, Hofman, and Loeys (2011) reasoned that this variable might interact with how a sentence context influences cross-lingual activation for homographs. They explicitly distinguished between interlingual homographs sharing the same word class and those that do not. For example, angel has the same word class as the Dutch reading's meaning sting, whereas breed is a verb or a noun and has a different word class from the Dutch reading's meaning wide. Baten et al. reasoned that the influence of word class might be particularly important when presenting words in a sentence context in which word meaning and sentence constraint interact. DutchEnglish bilinguals performed an L2 (English) lexical decision task to target words appearing as final words in a sentence. Both the homograph and its control word could appear in the same low-constraint sentence (e.g., She looked up and there seemed to be an angellalien: where angel is the homograph and alien is the control). Reaction times for homographs were faster than for controls when the two readings of the interlingual homograph had the same word class, but this homograph facilitation was eliminated when there was no such word class overlap. So the overlap in orthographic representation for homographs only led to faster processing times in sentence context for homographs that had the same word class. These results suggest that the presence of a sentence context indicating the word class of upcoming words can have a direct impact on cross-lingual activation. That is, only orthographic overlap for homographs did not lead to cross-lingual activation effects in a sentence context, but orthographic and word class overlap did.

Overall, these studies using tasks that require overt responses converge on the conclusion that the degree of language-nonselective activation is influenced by the semantic constraint of a sentence because a high-constraint, but not a low-constraint sentence context affected lexical access in bilinguals. However, even though the presentation of words in a sentence provides a more natural reading situation than word recognition out-of-context, the procedure still requires a response from the participant (e.g., a word/nonword response in lexical decision), which is not necessary in natural reading. Moreover, studies often presented target words at the end of a sentence context (e.g., Baten et al., 2011; Elston-Güttler et al., 2005). Sentencefinal words are typically read more slowly than sentence-internal words (e.g., Just \& Carpenter, 1980), and this sentence wrap-up effect has traditionally been explained by integrative processing that occurs at the end of sentences (e.g., Rayner, Kambe, \& Duffy, 2000). These processes might interfere with cross-lingual activation processes. Recent studies therefore have used the eye-tracking paradigm, in which participants can read normally as in everyday life, and no overt task other than comprehension is required. The time-sensitive eye movement measures allow researchers to investigate the time course of lexical activation by dissociating several early (reflecting initial lexical access) and late reading time measures (reflecting higher-order processes; Rayner, 1998). Indeed, eye-tracking studies in the monolingual domain (e.g., Duffy, Kambe, \& Rayner, 2001; Rayner, Binder, \& Duffy, 1999) suggest that the degree of competition between multiple meanings of an ambiguous word (e.g., bank as a financial institution or as a river side) depends on the relative time course of their activation. The time course of meaning activation, 
in turn, is determined by the relative frequencies of the ambiguous word's meaning, and this activation can be modulated by a biasing context (e.g., Duffy et al., 2001). In the next section, we discuss how the use of eye movements has deepened our knowledge of sentence context effects on bilingual visual word recognition in L2 and in L1.

\section{Natural Reading and Eye-Tracking}

\section{L2 Processing}

Duyck et al. (2007) investigated how the linguistic context provided by a sentence can constrain language-nonselective access in normal second language reading while measuring eye movements. The cognate facilitation effect was used as a marker of cross-lingual interactions because cognate effects have been shown to be strong and reliable in out-of-context studies (e.g., Dijkstra et al., 1999; Van Hell \& Dijkstra, 2002). Duyck et al. selected both identical (e.g., Dutch-English: ring) and nonidentical cognates (e.g., schip-ship) to examine how cross-linguistic overlap between translation equivalents may interact with the cognate effect in sentence context. In Experiment 1, the L2 cognate facilitation effect, as found in earlier single-word studies (e.g., Dijkstra et al., 1999; Lemhöfer \& Dijkstra, 2004), was replicated. Proficient Dutch-English bilinguals were presented with cognates, matched control words, filler words, and nonwords in an L2 (English) lexical decision task out-of-context. Reaction times were faster for cognates than for controls, and this effect interacted with the degree of cross-linguistic overlap: cognate facilitation was stronger for identical than for nonidentical cognates. This experiment validated the stimulus materials for use in the sentence studies.

In the second experiment, the same cognates and controls were presented as the final words of low-constraint sentences (presented word by word using RSVP), to which an L2 lexical decision had to be made. Both the cognate and its matched noncognate fit the same sentence (e.g., Hilda was showing off her new ring/coat; ring is the cognate; coat is the control). Similar to the presentation out-of-context, a cognate facilitation effect was obtained, and this effect was stronger for identical than for nonidentical cognates. This finding again shows that the unilingual linguistic context provided by a sentence does not eliminate cross-lingual interactions (cf. Schwartz \& Kroll, 2006; Van Hell \& De Groot, 2008).

The third experiment presented the cognates and controls in the middle of L2 low-constraint sentences while measuring eye movements. The eye-tracking technique can investigate reading in its most natural way in a laboratory situation and can distinguish between several early and late reading time measures. Early measures typically include first fixation duration (i.e., the duration of the first fixation on the target word) and gaze duration (i.e., the sum of fixations from the moment the eyes land on the target until they move off again). Late measures typically include go-past time (i.e., the time elapsing from encountering a target for the first time until 
a region to the right of the target is fixated), which also takes into account regressions originating from the target. The results showed cognate facilitation effects on the reading times for identical cognates on first fixations from $249 \mathrm{~ms}$ onwards after first encountering the word and also on later go-past time. Such cognate facilitation was not present for nonidentical cognates. This result indicates that the amount of cross-lingual activation is a function of the similarity between the translation equivalents. A sentence context providing a language cue might eliminate L2 cognate effects when cross-lingual activation is weaker (i.e., nonidentical cognates), but not when overlap is complete (i.e., identical cognates). Furthermore, the fact that nonidentical cognate effects in low-constraint sentences were observed in lexical decision but not in normal reading, as measured via eye movements, indicates that context and lexical variables (i.e., the degree of cross-lingual overlap between translation equivalents) may also interact with task-specific factors.

Libben and Titone (2009) later showed that even in a high-constraint sentence context, lexical activation is initially language-nonselective, although previous studies using tasks requiring overt responses (e.g., lexical decision; see for example, Schwartz \& Kroll, 2006; Van Hell \& De Groot, 2008) suggested that a semantically constraining sentence can constrain lexical selection to the target language. They presented French-English identical cognates (e.g., divorce) and interlingual homographs (e.g., chat: cat in French) in L2 (English) sentences that were either low or high in terms of semantic constraint for the target (e.g., high-constraint homograph sentence: Since they like to gossip, they had an extended chat that lasted all night; control sentence: Since they liked to compose songs, he made an extended tune that was very catchy). Highly proficient French-English bilinguals read the sentences while eye movements were measured. The results of early reading time measures (e.g., first fixation, gaze duration) revealed that homographs were read more slowly than matched controls in both low- and high-constraint sentences. Cognate facilitation was present on early reading time measures in low- and highconstraint sentences.

Thus, lexical access was nonselective and not modulated by semantic constraint in approximately the first $350 \mathrm{~ms}$ upon fixating the word. However, in the time range of approximately $350-600 \mathrm{~ms}$ of later reading time measures (e.g., go-past time), cognate facilitation and homograph inhibition was still present in lowconstraint sentences, but not any longer in high-constraint sentences. Libben and Titone (2009) suggested that lexical access is initially language-nonselective, but that this cross-language activation is nullified by top-down factors such as semantic constraint of a sentence at later word processing stages. The absence of cognate facilitation in high-constraint sentences for later stage results (e.g., go-past time) is consistent with the lexical decision results of Schwartz and Kroll (2006) and naming results of Van Hell and De Groot (2008) and suggests that these tasks may reflect comprehension processes occurring after lexical access had taken place. Furthermore, Libben and Titone also suggested that the absence of homograph interference effects in Schwartz and Kroll (2006) may be related to task characteristics. RSVP and word naming may be less sensitive than eye-tracking to detect cross-lingual interference effects. 
The absence of cross-lingual activation effects at later stages of comprehension in semantically constraining sentences reported in Libben and Titone (2009) contrasts with the results of Van Assche, Drieghe, Duyck, Welvaert, and Hartsuiker (2011), who did observe dual-language activation on late eye movement measures. This difference may originate from the fact that the Dutch-English bilinguals in Van Assche et al. were less balanced than the bilinguals tested in Libben and Titone. This may lead to stronger L1 activation in the bilinguals tested in Van Assche et al. (2011). Indeed, Titone, Libben, Mercier, Whitford, and Pivneva (2011) suggested that the bilinguals in Van Assche et al. may have experienced greater L1-to-L2 cross-language activation, so that semantic context may be insufficient to diminish cross-language activation.

The Dutch-English bilinguals in Van Assche et al. read cognates and matched control words in low and high semantically constrained sentences in their L2 while eye movements were recorded (e.g., low-constraint cognate sentence: He went to the shop to buy a book that he needed for school; low-constraint control sentence: She did not want to look at her face while she was crying). Cognate facilitation was shown on early and late eye movement measures, both for low- and high-constraint sentences. Moreover, facilitation increased gradually as a function of cross-lingual overlap between translation equivalents: higher orthographic overlap between translation equivalents on Van Orden's (1987) word similarity measure for cognates and controls led to faster reading times. These results indicate that semantic constraint does not affect cross-lingual activation in the bilingual language system at any stage of word recognition.

The cognate eye-tracking results in semantically constraining sentences in Van Assche et al. (2011) and Libben and Titone (2009) contrast with the previous studies of Schwartz and Kroll (2006) and Van Hell and De Groot (2008) who observed no cognate facilitation on lexical decision and naming times in high-constraint sentences. Van Assche et al. tested whether this difference between studies may be related to the different methodology used in an additional experiment, in which the stimulus materials of Van Assche et al. were presented using the paradigm of Van Hell and De Groot. They observed a weak cognate facilitation effect in highconstraint sentences, and this effect only emerged after running many more participants than did Van Hell and De Groot. These findings illustrate that the eye-tracking paradigm may be more sensitive to detecting cross-lingual activation effects than tasks requiring overt responses.

Balling (2012) recently tested an even more natural reading situation than word recognition in sentences. She had Danish-English bilinguals read cognates in texts or paragraphs. Cognate facilitation was observed that was modulated by morphological complexity. There was cognate facilitation for simple cognates (e.g., DanishEnglish rolle-role). This observation extends the evidence for language-nonselective access for word recognition in sentences to reading in texts. There was also an inhibitory effect for complex cognates (e.g., words that contain at least one cognate morpheme as in onsdag-Wednesday, where dag-day is the cognate morpheme). Balling suggested that problems in the integration of cognate and noncognate morphemes might lead to this inhibition. 
Note that the above studies all used noun stimuli to investigate languagenonselective activation in bilinguals and that theoretical accounts of bilingual language processing (e.g., Dijkstra \& Van Heuven, 2002) are almost exclusively based on noun processing. Van Assche, Duyck, and Brysbaert (2013) therefore examined lexical access for verbs during sentence reading in $\mathrm{L} 2$ with Dutch-English bilinguals. Although verbs have generally smaller degrees of formal and semantic overlap between languages than nouns (Gentner, 1981), there was cognate facilitation for cognate and control verbs presented out-of-context and cognate facilitation remained on a late reading time measure (go-past time) when targets were presented in low semantically constraining sentences. Early reading time measures did not show cross-lingual activation effects though. Thus, although cross-lingual activation effects for verbs were weaker than for nouns, these results show that crosslingual activation is strong enough for verb cognate effects to arise.

\section{L1 Processing}

As in the literature on bilingual word recognition out-of-context, the majority of published sentence context studies have focused on L2 processing. Influences of L1 on L2 processing are indeed generally stronger than influences of L2 on L1 processing (e.g., Duyck, 2005; Haigh \& Jared, 2007; Jared \& Kroll, 2001) and so cross-lingual activation effects are more likely to be observed for L2 processing. However, in order to demonstrate the existence of a profoundly language-nonselective bilingual language system, influences of the weaker L2 on reading in the dominant language should be investigated. Van Hell and Dijkstra (2002) were the first to show cognate facilitation effects in L1, indicating that the bilingual's L2 knowledge influenced native-language reading. Van Assche et al. (2009) replicated this cognate facilitation effect for words out-of-context and then tested how language information of a sentence context may influence this cross-lingual activation effect. Dutch-English bilinguals read L1 low-constraint sentences which contained a cognate or a control word (e.g., Bert heeft een oude oven/lade gevonden tussen de rommel op zolder: "Bert has found an old oven/drawer among the rubbish in the attic"). Early reading time measures (i.e., first fixation duration) were shorter for cognates than for controls. Moreover, cognate facilitation was shown to be a continuous effect because cognate facilitation gradually increased as a function of cross-lingual similarity. The results show that the mere presentation of words in a sentence context does not restrict cross-lingual interaction effects in bilinguals during native-language reading. This indicates a limited role for topdown lexical restrictions generated by sentences on the cross-lingual activation in the bilingual lexicon.

Titone et al. (2011) investigated whether semantic constraint would modulate cross-language activation during native-language reading. In Experiment 1, they measured the eye movements of English-French bilinguals reading identical cognates (e.g., English-French: divorce) and interlingual homographs (e.g., chat: cat in French) in low- and high-constraint L1 sentences (e.g., high-constraint cognate sentence: Because of the bitter custody battle over the kids, the expensive divorce was 
a disaster vs. high-constraint control sentence: Because the maid of honor and best man were late, the expensive wedding was a disaster). Cognate facilitation was present on early reading time measures in both low- and high-constraint sentences, but this effect was modulated by age of L2 acquisition: only bilinguals who acquired their L2 early in life showed cognate facilitation. Age of L2 acquisition did not modulate cognate effects on late reading time measures, but here, semantic constraint did: cognate facilitation was smaller in high- than in low-constraint sentences. There were no early homograph interference effects. Homograph interference was only present on total reading times and, contrary to the L2 results of Libben and Titone (2009), was unaffected by the semantic constraint of the sentence.

In the second experiment, L2 (French) filler sentences were intermixed with the experimental English sentences to examine whether making the L2 more salient would increase cognate and homograph effects during L1 reading. Indeed, under these experimental conditions, cognate facilitation was not reduced. The inclusion of L2 filler sentences seems to have increased cross-lingual activation during L1 sentence reading, and this process may have counteracted the semantic constraint effect. Homograph interference was present on total reading times, and this effect was stronger in Experiment 2 than in Experiment 1.

\section{Theoretical Accounts}

Although cognate and homograph effects have often been taken as evidence for an integrated lexicon in which words from both languages are represented and/or for lexical access of words from both languages in parallel, the precise representation of cognates and homographs and the modeling of task and context effects is a strongly debated topic (cf. Costa, Santesteban, \& Cano, 2005; Dijkstra et al., 2010; see Van Assche et al., 2012 and Degani \& Tokowicz, 2010, for reviews). A theoretical explanation of the cross-language activation effects discussed above can be given within bilingual language processing models such as the Bilingual Interactive Activation Plus Model (BIA+; Dijkstra \& Van Heuven, 2002) and a bilingual extension of the Re-ordered Access Model of Duffy, Morris, and Rayner (1988; Arêas Da Luz Fontes \& Schwartz, 2010; Degani \& Tokowicz, 2010).

The BIA+ model (Dijkstra \& Van Heuven, 2002) assumes that L1 and L2 words are represented in an integrated lexicon and that representations from both languages become activated in parallel. Lexical representations are activated depending on the overlap with the input stimulus and the resting level activation of the representations (based on frequency, proficiency, etc.). Cognates have similar orthographic and phonological representations and the same semantic representation. This high degree of similarity across languages speeds up their activation and recognition, as compared to noncognates. However, other theoretical accounts of the cognate facilitation effect assume qualitative differences in the representation of cognates and noncognates at a conceptual (e.g., De Groot \& Nas, 1991) or a morphological level (e.g., Sánchez-Casas \& García-Albea, 2005; see e.g., De Groot, 
2011; Dijkstra et al., 2010, for an overview). Interlingual homographs, on the other hand, have different semantic representations but they have the same orthographic representations in both languages. Control words only activate representations in one language. This difference in activation levels gives rise to the homograph effect.

Another theoretical account of cross-lingual homograph effects is an extension of the monolingual Re-ordered Access Model of Duffy, Morris, and Rayner (1988; Arêas Da Luz Fontes \& Schwartz, 2010; see also Degani \& Tokowicz, 2010; Schwartz \& Van Hell, 2012). According to this monolingual model, the extent to which each meaning of a homonym (e.g., bank as a riverside or a financial institution) is activated depends on the relative frequency of the meanings and on the syntactic/semantic context biasing a certain meaning. For instance, for homonyms presented without a biasing context, the relative frequency of the meanings determines the time course of their activation. A strong biasing context can reorder this activation. For the bilingual case, Arêas Da Luz Fontes and Schwartz (2010) propose that, in addition to frequency and context, cross-language activation may influence the time course of meaning activation. All three factors can interact with each other to activate the meaning of interlingual homographs in each language and therefore affect cross-lingual homograph effects.

In the BIA+ model, language membership is represented via language nodes such that all words from the same language are connected to a corresponding language node. The language nodes also reflect the global activity of each language. In the earlier BIA model (Dijkstra \& Van Heuven, 1998), the language nodes could suppress the activation of words in the other language through inhibition mechanisms. Later, in the BIA+ model, the language nodes served only a representational function; they can be pre-activated by the sentence, but they cannot influence the activation of words in the other language. As such, Dijkstra and Van Heuven (2002) predicted that the mere presentation of words in a sentence does not constrain language-nonselective activation. Indeed, the fact that cross-lingual activation effects were preserved in low-constraint sentences in L2 (e.g., Libben \& Titone, 2009; Schwartz \& Kroll, 2006; Van Assche et al., 2011; Van Hell \& De Groot, 2008) and in L1 (e.g., Titone et al., 2011; Van Assche et al., 2009) provides support for the assumption of limited influence of the language of the sentence on languagenonselective activation.

Turning to the effect of semantic constraint on lexical activation, Dijkstra and Van Heuven (2002) suggested that syntactic and semantic context might directly affect the word identification system. This may change the degree of languagenonselectivity in bilingual word recognition in a similar way, as sentence context influences monolingual word recognition (e.g., Schwanenflugel \& LaCount, 1988). Indeed, lexical decision and naming studies have revealed that a semantic context could constrain lexical access (e.g., Schwartz \& Kroll, 2006; Van Hell \& De Groot, 2008), but eye-tracking studies did not find the same results (e.g., Van Assche et al., 2011) or found an effect of semantic constraint only in later processing stages (e.g., Libben \& Titone, 2009). This suggests that the semantic context effect on lexical activation may occur during later stages of word recognition, although not all studies support this suggestion (Van Assche et al., 2011). 
In order to account for differences between experiments and nonlinguistic context effects (e.g., task characteristics, participant's expectations) in the BIA+ model, a distinction is made between the word identification system containing orthographic, phonological, and semantic representations and the task/decision system, analogous to Green (1998). This additional task/decision system allows distinguishing processes that influence the activation of lexical representations in the word identification system from processes that influence participants' decision criteria. Cross-experimental differences are thus handled by the task/decision system affecting the output of the word identification system. Dijkstra and Van Heuven (2002) propose that nonlinguistic information affects only the decision criteria related to task demands rather than the activation level of lexical representations in the two languages (for more information, see Dijkstra \& Van Heuven, 2002).

\section{Creating a Sentence Context Experiment}

The studies presented above illustrate that the design of an experiment and its specific task choice or stimulus materials can influence the results and subsequent conclusions substantially. In this section, we discuss the procedures and points of interest for designing a sentence context experiment using the eye-tracking paradigm.

\section{Participants}

In selecting the bilinguals to take part in the experiment, it is important to carefully consider several factors that have been shown to influence cross-language activation effects in visual word and sentence processing such as L2 (and L3) proficiency (e.g., Van Hell \& Dijkstra, 2002) and age of L2 acquisition (e.g., Titone et al., 2011). Bilinguals are often asked to rate their speaking, reading, writing, and comprehension abilities in each language as a measure of proficiency in language history questionnaires, even though the validity of these questionnaires has only rarely been tested. Recent studies explicitly addressed this issue, and this has led to the development of validated instruments such as the Language Experience and Proficiency Questionnaire (LEAP-Q, Marian, Blumenfeld, \& Kaushanskaya, 2007). There are also more direct tests of proficiency. The LexTALE is a short lexical test for advanced learners of English as an L2 and has been shown to be a good predictor of English vocabulary knowledge and general English proficiency (Lemhöfer \& Broersma, 2012). A similar test to measure language proficiency in French has been developed by Brysbaert (2013). Another recent test to measure language dominance and language proficiency in spoken production is the Multilingual Naming Test (MINT; Gollan, Weisberger, Runnqvist, Montoya, \& Cera, 2012). A paper-andpencil dominance scale to quantify the language dominance of bilinguals was presented by Dunn and Fox Tree (2009). 


\section{Stimulus Materials}

Selection of stimulus materials can often be a time-consuming process in language research because word and sentence stimuli have to be carefully selected and controlled on a number of factors. For instance, testing cognates or homographs in a factorial design (i.e., comparing processing of the set of cognates with the set of control words) requires the selection of control words that are matched to the cognates on word characteristics such as word length, word frequency, and number of orthographic neighbors (Coltheart et al., 1977). These factors have been shown to significantly influence word processing (e.g., New et al., 2006). The WordGen program (Duyck et al., 2004) can be used to calculate the values of these variables for selected words in Dutch, English, German, and French. It can also be used to select control words and to generate nonwords (for use in a lexical decision task) adhering to any combination of linguistic constraints such as number of letters, neighborhood size, frequency, and summated bigram frequency. Another example of a resource for psycholinguistic research is LexicALL that contains useful datasets such as Chinese, Dutch, and English word frequencies based on film and television subtitles (e.g., Cai \& Brysbaert, 2010; Cuetos, Glez-Nosti, Barbon, \& Brysbaert, 2011). It also includes Wuggy, which is a multilingual generator of nonwords (Keuleers \& Brysbaert, 2010). More information on LexicALL and Wuggy can be found on www.crr.ugent.be (see Related Internet Sites).

Selection of stimulus materials does not necessarily have to include the selection of cognates and homographs and matched controls. Cross-lingual overlap can also be investigated as a continuous measure (see Van Assche et al., 2009, 2011). For instance, orthographic overlap of a set of stimulus materials including cognates and noncognates can be calculated using the Van Orden (1987) word similarity measure or Levenshtein distance (Levenshtein, 1966). By calculating an orthographic overlap score for each translation word pair (e.g., Dutch-English: schouder-shoulder has a Van Orden overlap value of .81; leraar-teacher of .30; for more information on the calculation of Van Orden overlap scores, see Related Internet Sites), it can be investigated whether more orthographic overlap across languages facilitates word processing. These measures do not take into account phonological overlap, and therefore, additional ratings will have to be collected.

In a sentence context experiment including a constraint manipulation, low- and high-constraint sentences have to be created for each target word and matched control. Sentences for targets and controls are preferably matched on number of words, syntactic structure, and the length of the word preceding the target. Critical words cannot be presented as the final word of the sentence because of sentence wrap-up processes on sentence-final positions (Rayner et al., 2000). To qualify sentences as low or high in terms of semantic constraint, sentence completion ratings have to be collected in a separate cloze probability study, in which participants are presented with the sentence frames up to the target word. They are instructed to write down the first word that comes to mind when reading the sentence. In order for the constraint manipulation to be successful, high-constraint sentences should be completed with one specific word, whereas low-constraint sentences should be 
completed with a variety of words. The resulting cloze probabilities allow one to verify the constraint manipulation and to further optimize the sentences. In order to avoid having the participants see the same target word twice, sentences can be divided across two presentation lists, so that each participant sees the target word and its control in either the low- or high-constraint sentence context.

Even though stimulus materials are carefully controlled, it is always useful to conduct a control experiment with monolinguals if possible, to ensure that effects are not due to any uncontrolled stimulus characteristics. A control experiment consists of testing a group of participants who have no knowledge of the nontarget language on the same stimulus materials. These participants should not be influenced by cross-linguistic overlap.

\section{Procedure}

An eye-tracking experiment typically starts with camera setup and calibration. After calibration is completed, the instructions are given to the participants. They are instructed to read the sentences as naturally as possible for comprehension (as if one were reading a book or a newspaper). Sentences are presented as a whole on the screen and participants can press a button indicating that they have finished reading the sentences. For single-sentence experiments, it is advisable to display the sentence on no more than two lines and in monospaced Courier font. If a sentence has to be presented on two lines, make sure that target words are never the final word of a line, nor the first word of the second line. Comprehension of the sentences and attention to the reading task are typically examined by presenting comprehension questions following some trials. Participants can respond "Yes" or "No" to these questions using the appropriate buttons or keys. Sentences have to be presented in a random order to each participant. It is advisable to start with some practice sentences so that participants get used to the reading task.

\section{Summary and Conclusion}

The L2 and L1 studies on bilingual visual word recognition in sentence context show that the language of the preceding words is an insufficient cue to restrict lexical access to words of the target language (e.g., Duyck et al., 2007; Schwartz \& Kroll, 2006; Van Assche et al., 2009; Van Hell \& De Groot, 2008). This literature offers strong evidence for a bilingual language system that is profoundly languagenonselective. Furthermore, studies measuring eye movements revealed that the degree of semantic constraint for a sentence does not necessarily eliminate lexical activation of the nontarget language (Van Assche et al., 2011), although there is evidence that it has an effect that occurs relatively late (Libben \& Titone, 2009; Titone et al., 2011) and that it influences cross-lingual activation effects in lexical 
decision, translation, and naming studies (e.g., Schwartz \& Kroll, 2006; Van Hell \& De Groot, 2008). The difference in result patterns across studies indicates that the influence of a sentence and semantic context on language-nonselective activation is dependent on experimental factors such as task demands (e.g., lexical decision vs. eye-tracking; Van Assche et al., 2011; Van Hell \& De Groot, 2008), type of bilingual tested (e.g., proficiency and age of acquisition; Libben \& Titone, 2009; Van Assche et al., 2011), cross-overlap of translation equivalents (e.g., identical vs. nonidentical cognates; Duyck et al., 2007), and stimulus list composition (e.g., Titone et al., 2011).

\section{List of Keywords}

Bilingual Interactive Activation Plus Model (BIA+), Bilingual word recognition, Cognate facilitation effect, Cognates, Dual-language activation, Eye-tracking, Factorial design, First fixation duration, Gaze duration, Go-past time, Highconstraint sentence context, Higher-order processes, Homograph facilitation effect, Homographs, Initial lexical access, Interlingual homographs, Languagenonselective lexical access, Lexical access, Lexical decision task, Low-constraint sentence context, Naming task, Proficiency, Rapid serial visual presentation (RSVP), Re-ordered Access Model, Semantic priming effect

\section{Review Questions}

1. The studies presented in this chapter all involve bilinguals who speak languages with the same scripts. There are, however, also languages with completely different scripts (e.g., Chinese, Hebrew). How do you think cognates will be processed in these languages?

2. Think about the bilinguals living in your country. How proficient are they in both languages? Do they use both languages regularly? How do you think proficiency can influence the degree of language-nonselective activation in the bilingual language system?

3. Can you think of other tasks that can be used to investigate whether bilinguals activate words in one or both of their languages? What advantages or disadvantages can you think of for each task?

\section{Suggested Student Research Projects}

1. Textbook assignment. Choose a page in a magazine or newspaper. See whether you can find cognates. How many cognates were you able to find? Some languages share many words across languages while other language pairs do not, 
so depending on the relevant language pairs in your language context, you will find many or only a few. Check the word categories of the cognates. Most of the studies discussed in this chapter have focused on the processing of nouns. Do you think that the same results can be found for verbs or adjectives? In answering this question, especially consider the degree of semantic, orthographic, and phonological overlap between the words.

2. Creating a lexical decision experiment. In this project, you will try to find evidence for the hypothesis you generated above for cognate effects for other word categories, such as verbs or adjectives. Try to create your own lexical decision experiment in which you will examine whether cognates are processed more quickly than controls.

3. Semantic processing in experimental tasks. The processing of homographs in lexical decision tasks has shown that the orthographic overlap for homographs can lead to facilitation. However, how do you think homographs will be processed in tasks that obligatorily involve semantic processing (e.g., semantic categorization)?

\section{Related Internet Sites}

Experimental materials: http://www.tamiu.edu/ rheredia/materials.html LexicALL: Data-sets: http://lexicall.widged.com/repository/listing.php Software and data-sets: http://crr.ugent.be/

SUBTLEXus: Word frequency American English: http://expsy.ugent.be/subtlexus/ Van Orden overlap score: http://users.ugent.be/ rhartsui/Applet1.html Word generator: WordGEn: http://www.wouterduyck.be/?page_id=29 Word frequencies: http://crr.ugent.be/programs-data/subtitle-frequencies

Wuggy: Multilingual pseudoword generator: http://crr.ugent.be/programs-data/ wuggy

\section{Suggested Further Reading}

Degani, T., \& Tokowicz, N. (2010). Semantic ambiguity within and across languages: An integrative review. The Quarterly Journal of Experimental Psychology, 63, 1266-1303.

De Groot, A. M. B. (2011). Language and cognition in bilinguals and multilinguals: An introduction. New York: Psychology Press.

Dijkstra, T., \& Van Heuven, W. J. B. (2002). The architecture of the bilingual word recognition system: From identification to decision. Bilingualism: Language and Cognition, 5, 175-197.

Kroll, J.F., \& De Groot, A. M. B. (2005). Handbook of bilingualism: Psycholinguistic approaches. New York: Oxford University Press.

Van Assche, E., Duyck, W., \& Hartsuiker, R. J. (2012). Bilingual word recognition in a sentence context. Frontiers in Psychology, 3, 174. 


\section{References}

Altarriba, J., Kroll, J. F., Sholl, A., \& Rayner, K. (1996). The influence of lexical and conceptual constraints on reading mixed-language sentences: Evidence from eye fixations and naming times. Memory \& Cognition, 24, 477-492.

Arêas Da Luz Fontes, A. B., \& Schwartz, A. I. (2010). On a different plane: Cross-language effects on the conceptual representations of within-language homonyms. Language and Cognitive Processes, 25, 508-532.

Balling, K. W. (2012). Reading authentic texts: What counts as cognate. Bilingualism: Language and Cognition, 16(3), 637-653.

Baten, K., Hofman, F., \& Loeys, T. (2011). Cross-linguistic activation in bilingual sentence processing: The role of word class meaning. Bilingualism: Language and Cognition, 14, 351-359.

Bentin, S., Mouchetant-Rostaing, Y., Giard, M. H., Echalier, J. F., \& Pernier, J. (1999). ERP manifestations of processing printed words at different psycholinguistic levels: Time course and scalp distribution. Journal of Cognitive Neuroscience, 11, 235-260.

Brown, C. M., \& Hagoort, P. (1993). The processing nature of the N400: Evidence from masked priming. Journal of Cognitive Neuroscience, 5, 34-44.

Brysbaert, M. (2013). LEXTALE_FR: A fast, free, and efficient test to measure language proficiency in French. Psychologica Belgica, 53, 23-37.

Cai, Q., \& Brysbaert, M. (2010). SUBTLEX-CH: Chinese word and character frequencies based on film subtitles. PLoS One, 5, e10729.

Caramazza, A., \& Brones, I. (1979). Lexical access in bilinguals. Bulletin of the Psychonomic Society, 13, 212-214.

Coltheart, M., Davelaar, A., Jonasson, J. T., \& Besner, D. (1977). Access to the internal lexicon. In S. Dornic (Ed.), Attention and performance (Vol. 6, pp. 535-555). New York: Academic.

Costa, A., Santesteban, M., \& Cano, A. (2005). On the facilitatory effects of cognates in bilingual speech production. Brain and Language, 94, 94-103.

Cuetos, F., Glez-Nosti, M., Barbon, A., \& Brysbaert, M. (2011). SUBTLEX-ESP: Spanish word frequencies based on film subtitles. Psicológica, 32, 133-143.

De Groot, A. M. B. (2011). Language and cognition in bilinguals and multilinguals: An introduction. New York: Psychology Press.

De Groot, A. M. B., \& Nas, G. L. J. (1991). Lexical representations of cognates and noncognates in compound bilinguals. Journal of Memory and Language, 30, 90-123.

Degani, T., \& Tokowicz, N. (2010). Semantic ambiguity within and across languages: An integrative review. The Quarterly Journal of Experimental Psychology, 63, 1266-1303.

Dijkstra, T., Grainger, J., \& Van Heuven, W. J. B. (1999). Recognition of cognates and interlingual homographs: The neglected role of phonology. Journal of Memory and Language, 41, 496-518.

Dijkstra, T., Miwa, K., Brummelhuis, B., Sappelli, M., \& Baayen, H. (2010). How cross-language similarity and task demands affect cognate recognition. Journal of Memory and Language, 62, 284-301.

Dijkstra, T., Timmermans, M., \& Schriefers, H. (2000). On being blinded by your other language: Effects of task demands on interlingual homograph recognition. Journal of Memory and Language, 42, 445-464.

Dijkstra, T., \& Van Heuven, W. J. B. (1998). The BIA model and bilingual word recognition. In J. Grainger \& A. Jacobs (Eds.), Localist connectionist approaches to human cognition (pp. 189-225). Mahwah, NJ: Lawrence Erlbaum.

Dijkstra, T., \& Van Heuven, W. J. B. (2002). The architecture of the bilingual word recognition system: From identification to decision. Bilingualism: Language and Cognition, 5, 175-197.

Dijkstra, T., Van Jaarsveld, H., \& Ten Brinke, S. (1998). Interlingual homograph recognition: Effects of task demands and language intermixing. Bilingualism: Language and Cognition, 1, $51-66$. 
Duffy, S. A., Kambe, G., \& Rayner, K. (2001). The effect of prior disambiguating context on the comprehension of ambiguous words: Evidence from eye movements. In D. S. Gorfein (Ed.), On the consequences of meaning selection: Perspectives on resolving lexical ambiguity. Decade of behavior (pp. 27-44). Washington, DC: American Psychological Association.

Duffy, S. A., Morris, R. K., \& Rayner, K. (1988). Lexical ambiguity and fixation times in reading. Journal of Memory and Language, 27, 429-446.

Dunn, A. L., \& Fox Tree, J. E. (2009). A quick, gradient Bilingual Dominance Scale. Bilingualism: Language and Cognition, 12, 273-289.

Duyck, W. (2005). Translation and associative priming with cross-lingual pseudohomophones: Evidence for nonselective phonological activation in bilinguals. Journal of Experimental Psychology: Learning, Memory, and Cognition, 31, 1340-1359.

Duyck, W., Desmet, T., Verbeke, L. P. C., \& Brysbaert, M. (2004). WordGen: A tool for word selection and nonword generation in Dutch, English, German, and French. Behavior Research Methods, Instruments \& Computers, 36, 488-499.

Duyck, W., Van Assche, E., Drieghe, D., \& Hartsuiker, R. J. (2007). Visual word recognition by bilinguals in a sentence context: Evidence for nonselective lexical access. Journal of Experimental Psychology: Learning, Memory, and Cognition, 33, 663-679.

Elston-Güttler, K. E., Gunter, T. C., \& Kotz, S. A. (2005). Zooming into L2: Global language context and adjustment affect processing of interlingual homographs in sentences. Cognitive Brain Research, 25, 57-70.

Gentner, D. (1981). Some interesting differences between verbs and nouns. Cognition and Brain Theory, 4, 161-178.

Gollan, T. H., Weisberger, G. H., Runnqvist, E., Montoya, R. I., \& Cera, C. M. (2012). Self-ratings of spoken language dominance: A Multilingual Naming Test (MINT) and preliminary norms for young and aging Spanish-English bilinguals. Bilingualism: Language and Cognition, $15(3), 594-615$.

Green, D. W. (1998). Mental control and the bilingual lexico-semantic system. Bilingualism: Language and Cognition, 1, 67-81.

Haigh, C., \& Jared, D. (2007). The activation of phonological representations by bilinguals while reading silently: Evidence from interlingual homophones. Journal of Experimental Psychology: Learning Memory and Cognition, 33(4),623-44.

Jared, D., \& Kroll, J. F. (2001). Do bilinguals activate phonological representations in one or both of their languages when naming words? Journal of Memory and Language, 44, 2-31.

Just, M. A., \& Carpenter, P. A. (1980). A theory of reading: From eye fixations to comprehension. Psychological Review, 87, 329-354.

Keuleers, E., \& Brysbaert, M. (2010). Wuggy: A multilingual pseudoword generator. Behavior Research Methods, 42, 627-633.

Lemhöfer, K., \& Broersma, M. (2012). Introducing LexTALE: A quick and valid Lexical Test for Advanced Learners of English. Behavior Research Methods, 44, 325-343.

Lemhöfer, K., \& Dijkstra, T. (2004). Recognizing cognates and interlingual homographs: Effects of code similarity in language-specific and generalized lexical decision. Memory and Cognition, $32,533-550$.

Lemhöfer, K., Dijkstra, T., \& Michel, M. C. (2004). Three languages, one echo: Cognate effects in trilingual word recognition. Language and Cognitive Processes, 19, 585-611.

Levenshtein, V. I. (1966). Binary codes capable of correcting deletions, insertions and reversals. Soviet Physics Doklady, 10, 707.

Libben, M. R., \& Titone, D. A. (2009). Bilingual lexical access in context: Evidence from eye movements during reading. Journal of Experimental Psychology: Learning, Memory, and Cognition, 35, 381-390.

Marian, V., Blumenfeld, H. K., \& Kaushanskaya, M. (2007). The language experience and proficiency questionnaire (LEAP-Q). Journal of Speech, Language, and Hearing Research, 50, 940-967. 
New, B., Ferrand, L., Pallier, C., \& Brysbaert, M. (2006). Reexamining the word length effect in visual word recognition: New evidence from the English lexicon project. Psychonomic Bulletin \& Review, 13, 45-52.

Rayner, K. (1998). Eye movements in reading and information processing: 20 years of research. Psychological Bulletin, 124, 372-422.

Rayner, K., Binder, K. S., \& Duffy, S. A. (1999). Contextual strength and the subordinate bias effect: Comment on Martin, Vu, Kellas, and Metcalf. Quarterly Journal of Experimental Psychology, 52, 841-852.

Rayner, K., Kambe, G., \& Duffy, S. A. (2000). The effect of clause wrap-up on eye movements during reading. The Quarterly Journal of Experimental Psychology, 53, 1061-1080.

Sánchez-Casas, R. M., \& García-Albea, J. E. (2005). The representation of cognate and noncognate words in bilingual memory: Can cognate status be characterized as a special kind of morphological relation? In J. F. Kroll \& A. M. B. De Groot (Eds.), Handbook of bilingualism: Psycholinguistic approaches (pp. 226-250). Oxford, England: Oxford University Press.

Schwanenflugel, P. J., \& LaCount, K. L. (1988). Semantic relatedness and the scope of facilitation for upcoming words in sentences. Journal of Experimental Psychology: Learning, Memory, and Cognition, 14, 344-354.

Schwartz, A. I., \& Kroll, J. F. (2006). Bilingual lexical activation in sentence context. Journal of Memory and Language, 55, 197-212.

Schwartz, A. I., \& Van Hell, J. (2012). Bilingual visual word recognition. In J. Adelman (Ed.), Visual word recognition (pp. 131-150). New York: Psychology Press.

Segui, J., \& Grainger, J. (1990). Priming word recognition with orthographic neighbors: Effects of relative prime-target frequency. Journal of Experimental Psychology: Human Perception and Performance, 16, 65-76.

Stanovich, K. E., \& West, R. F. (1983). On priming by a sentence context. Journal of Experimental Psychology: General, 112, 1-36.

Titone, D., Libben, M., Mercier, J., Whitford, V., \& Pivneva, I. (2011). Bilingual lexical access during L1 sentence reading: The effects of L2 knowledge, semantic constraint, and L1-L2 intermixing. Journal of Experimental Psychology: Learning, Memory, and Cognition, 37, 1412-1431.

Van Assche, E., Drieghe, D., Duyck, W., Welvaert, M., \& Hartsuiker, R. J. (2011). The influence of semantic constraints on bilingual word recognition during sentence reading. Journal of Memory and Language, 64, 88-107.

Van Assche, E., Duyck, W., Hartsuiker, R. J., \& Diependaele, K. (2009). Does bilingualism change native-language reading? Cognate effects in a sentence context. Psychological Science, 20, 923-927.

Van Assche, E., Duyck, W., \& Brysbaert, M. (2013). Verb processing by bilinguals in sentence contexts: The effect of cognate status and verb tense. Studies in Second Language Acquisition, $35,1-23$.

Van Assche, E., Duyck, W., \& Hartsuiker, R. J. (2012). Bilingual word recognition in a sentence context. Frontiers in Psychology, 3, 174.

Van Hell, J. G., \& De Groot, A. M. B. (2008). Sentence context modulates visual word recognition and translation in bilinguals. Acta Psychologica, 128, 431-451.

Van Hell, J. G., \& Dijkstra, T. (2002). Foreign language knowledge can influence native language performance in exclusively native contexts. Psychonomic Bulletin \& Review, 9, 780-789.

Van Orden, G. C. (1987). A rows is a rose: Spelling, sound, and reading. Memory \& Cognition, 15, 181-198. 